\title{
INVARIANT SUBSPACES WITH INVARIANT COMPLEMENTS
}

\author{
C. F. SCHUBERT 1
}

1. Introduction. Let $H_{n}$ denote the Hardy class of functions in $H^{2}$ of the unit disk $\Delta$ with values in the complex Hilbert space $C_{n}$. If $z: H_{n} \rightarrow H_{n}$ denotes the operator of multiplication by $z$ and $z^{*}$ is its operator conjugate on $H_{n}$, then one consequence of Theorem 1 of [3] is that the only projection ${ }^{2} P$ on $H_{n}$ which commutes with both $z$ and $z^{*}$ can be represented as a constant $n \times n$ matrix $P=\left[P_{i j}\right]$ which acts on $H_{n}$ in the following way: if $u=\left\{u_{j}\right\}_{j=1}^{n} \in H_{n}$ then $P u=\left\{\sum_{j} P_{i j} u_{j}\right\}_{i=1}^{n}$. An alternative interpretation is that the only orthogonal projection $P$ on $H_{n}$ which commutes with $z$ is necessarily of the above form where $\left[P_{i j}\right]$ is an orthogonal projection on $C_{n}$. While orthogonal projection is the natural projection in Hilbert space it is still only one of many and we examine here the class of projections on $H_{n}$ which commute with $z$ but not necessarily with $z^{*}$. In $H_{1}\left(=H^{2}\right)$ essentially nothing new happens. The consequences of Theorem 1 of [3] quoted above imply that if $P$ is a projection on $H_{1}$ which commutes with $z$ and $z^{*}$ then $P=0$ or $I$. The same is true even if $P$ merely commutes with $z$ e.g. [4, Problem 116]. In $\mathrm{H}_{2}$ things are different. If $P$ denotes the matrix

$$
\left(\begin{array}{cc}
z, & z \\
1-z, & 1-z
\end{array}\right)
$$

then $P$ is a projection on $H_{2}$ which commutes with $z$, but is not a matrix of constants. The projection $P$ decomposes $H_{2}$ into the direct sum $S \oplus T$ of two invariant subspaces

$$
\left.S=\left\{\left(u_{1}, u_{2}\right)=(z w,(1-z) w) \mid w \in H^{2}\right)\right\}
$$

and

$$
T=\left\{\left(u_{1}, u_{2}\right)=(w,-w) \mid w \in H^{2}\right\} .
$$

The problem of determining the projections on $H_{n}$ which commute with $z$ is equivalent to the problem: Determine all translation invariant subspace $S$ of $H_{n}$ which have a translation invariant comple-

Received by the editors September 10, 1968.

1 The preparation of this paper was supported in part by National Science Foundation grant no. GP-8922.

2 Unless stated otherwise, "projection" means "continuous projection" and "subspace" means "closed subspace." 
ment in $H_{n}$, i.e. subspaces $S$ such that $z S \subset S$ and $H_{n}=S \oplus T$ for some subspace $T$ satisfying $z T \subset T$. Call such a subspace $S$ invariantly complemented, or i.c. for brevity. The simplest nontrivial i.c. subspaces of $H_{n}$ are of the form $S=A H_{p}$ where $A$ is an $n \times p$ matrix of elements of $H^{\infty}$ which by the addition of $n-p$ columns can be made into an isomorphism of $H_{n}$ onto itself. In fact these are the only i.c. subspaces; see Theorem 2.1 below. The remaining results of this paper are extensions to matrices of elements from $H^{\infty}$, of some elementary properties of matrices with complex entries. One of these, the corollary following Theorem $2.2^{\prime}$, is a natural extension of the Corona Theorem differing slightly from that of Fuhrmann [2].

The significant results needed here are the Corona Theorem [1], Lax's characterization of the invariant subspaces of $H_{n}$ [5], [6], and the fact that any continuous linear operator $A: H_{n} \rightarrow H_{n}$ which commutes with $z$ is representable as an $n \times n$ matrix of elements of $H^{\infty}$ and $A$ operates on $H_{n}$ by matrix multiplication. Moreover if $A$ is a topological linear isomorphism of $H_{n}$ onto itself then $|\operatorname{det} A(z)|$ $\geqq \epsilon>0$ for some $\epsilon$ and all $z \in \Delta$.

To conclude this introduction we note a property of i.c. subspaces which will not be used later, but which has some independent interest. If $S$ is i.c. and $u \in H_{n}$ is such that $z u \in S$, then if $P$ is a projection onto $S$ commuting with $z, z u=P z u=z P u$, i.e. $z(u-P u)=0$ or $u=P u \in S$. This extends immediately to polynomials, i.e. if $p(z)$ is a polynomial and $u \in H_{n}$ is such that $p(z) u \in S$, then $u \in S$, and taking weak limits is true even if $p(z) \in H^{\infty}$, since the polynomials are dense in the weak star topology on $H^{\infty}$. This property is not shared by all invariant subspaces, e.g. if $S$ is the invariant subspace $z^{2} H^{2}$ of $H^{2}$ and $p(z)=z$, then $z \in H^{2}$ and $z^{2}=p(z) \cdot z \in S$ but $z \notin S$. There is some evidence to suggest that this property is characteristic of i.c. subspaces. The following lemma is a sample of such evidence.

Lemma 1.1. Let $\phi_{1}, \cdots, \phi_{n}$ be elements of $H^{\infty}$ such that $\sum\left|\phi_{j}\right|^{2}=1$ a.e. on $|z|=1$ and let $S \subset H_{n}$ be the invariant subspace of $H_{n}$, $\left\{\left(\phi_{1} u, \cdots, \phi_{n} u\right) \mid u \in H^{2}\right\} . A$ necessary and sufficient condition that for every Blashke product $p(z), u \in H_{n}$ and $p u \in S$ together imply $u \in S$, is that $\sum\left|\phi_{j}(z)\right|^{2}>0$ in $\Delta$.

The necessary and sufficient condition that $S$ be i.c. is that $\sum\left|\phi_{j}(z)\right|^{2}$ $\geqq \epsilon>0$ in $\Delta$, (Theorem 2.2 below). The above lemma uses only Blashke products and not the whole of $H^{\infty}$, but none the less is suggestive of the stronger result.

Proof of Lemma 1.1. Note first that it is sufficient to consider only all $p$ of the form $(z-a) /(1-\bar{a} z)$, for $|a|<1$. If $\sum\left|\phi_{j}(z)\right|^{2}>0$ and 
$u=\left(u_{1}, \cdots, u_{n}\right) \in H_{n}$ is such that $u_{i} p=\phi_{i} v$ for all $i$ and some $v \in H^{2}$ then $v(a)=0$ and so $p^{-1} v \in H^{2}$ whence $u \in S$ as required. Conversely, suppose that the $\phi_{j}$ have a common zero at $a \in \Delta$. Put $p(z)$ $=(z-a) /(1-\bar{a} z)$ and $u=\left(\phi_{1} / p, \cdots, \phi_{n} / p\right)$. Then $u \in H_{n}$ and $p u \in S$ but $u \notin S$, completing the proof.

2. Results. In all that follows we adopt the convention that if $S$ is a subspace of $H_{n}$ and $z \in \Delta, S(z)$ is the subspace of $C_{n}$ spanned by the numerical values of the elements of $S$ at the point $z$; in particular $H_{n}(z)=C_{n}$. We also identify $H_{p} \times H_{q}$ with $H_{p+q}$.

LEMMA 2.1. If there exists a continuous linear isomorphism $T$ of $H_{n}$ onto $H_{m}$ which commutes with $z$ then $m=n$.

Proof. Since $z T=T z, T$ can be represented as an $m \times n$ matrix of elements of $H^{\infty}$. By assumption $C_{m}=T(z) C_{n}$ for any $z \in \Delta$ and so $n \geqq m$. Similarly $T^{-1}$ as a map of $H_{m}$ onto $H_{n}$ being continuous by the closed graph theorem and also commuting with $z$, we have $n \leqq m$, and so $m=n$.

We now have our main theorem.

TheOREM 2.1. If $S \subset H_{n}$ is an i.c. subspace then there exists a linear isomorphism $A$ of $H_{n}$ onto itself such that $z A=A z$ and $S=A P_{p} H_{n}$ where $P_{p}$ denotes the projection of $H_{n}$ onto $H_{p}$ obtained by the coordinate projection $P_{p}\left\{u_{1}, \cdots, u_{n}\right\}=\left\{u_{1}, \cdots, u_{p}, 0, \cdots, 0\right\}$.

Proof. Since $S$ is i.c. there does exist a continuous projection $P$ of $H_{n}$ onto $S$ such that $z P=P z$ and $P$ may be realized as a matrix multiplication operator whose entries are elements of $H^{\infty}$. I-P also commutes with $z$ and projects $H_{n}$ onto a complementary subspace $T$. Both $S$ and $T$ are translation invariant and so by Lax's Theorem there exist $n \times p$ and $n \times q$ matrices $A_{S}$ and $A_{T}$ such that $A_{S}^{*}(z) A_{S}(z)$ $=I$ and $A_{T}^{*}(z) A_{T}(z)=I$ for almost all $z$ on $|z|=1$ and $S=A_{S} H_{p}$, $T=A_{T} H_{q}$. Here ${ }^{*}$ denotes the conjugate. In particular $A_{S}$ is invertible and $A_{S}^{-1}: S \rightarrow H_{p}$ is continuous and commutes with $z$. Similarly for $T$. Let $A: H_{p+q}=H_{p} \times H_{q} \rightarrow H_{n}$ be given by $A(u, v)=A_{S} u+A_{T} v$, where $u \in H_{p}, \quad v \in H_{q}$ and $B: H_{n} \rightarrow H_{p} \times H_{q}$ be given by $B u=A_{S}^{-1} P u$ $+A_{T}^{-1}(I-P) u$, then $A B=I, B A=I$ and $A$ is continuous and commutes with $z$. Thus by Lemma $2.1 p+q=n$, and identifying $H_{p+q}$ with $H_{n}$, we have $S=A_{S} H_{p}=A P_{p} H_{n}$ as asserted, and $A$ clearly commutes with $z$.

Since $A$ is invertible, so also is $A(z)$ and thus for all $z \in \Delta, \operatorname{dim} S(z)$ $=p$. We shall refer to this constant integer $p$, improperly, as $\operatorname{dim} S$. As further consequences of Theorem 2.1 we now have that $S=H_{n}$, if 
$S \subset H_{n}, S$ is i.c., and $\operatorname{dim} S=n$, cf. $H^{2}$ as mentioned in the introduction. Also the only irreducible i.c. subspaces of $H_{n}$ have dim 1 and are isomorphic to $H^{2}$.

In order to give an analytic characterization of i.c. subspaces we shall need the estimate of the following elementary lemma.

LemMa 2.2. Let $B$ be an invertible $n \times n$ matrix of complex numbers such that $\left|B_{i j}\right| \leqq \beta$. Suppose $A$ and $C$ are $n \times p(p<n)$ matrices such that $B A=C$. Then if $\left\{A^{k}\right\} \quad\left(\left\{C^{k}\right\}\right)$ denotes the set of all $p \times p$ submatrices of $A(C)$, we have

$$
\sum_{k}\left|\operatorname{det} C^{k}\right| \geqq \operatorname{det} B\left(\begin{array}{l}
n \\
p
\end{array}\right) \beta^{p-n}[(n-p) !]^{-1} \sum_{k}\left|\operatorname{det} A^{k}\right| .
$$

Proof. Choosing any one of the matrices $A^{k}$, augment the matrix $A$ by the addition, on the right, of $n-p$ columns each containing $n-1$ zeros and one one to obtain an $n \times n$ matrix $A^{(k)}$ such that $\left|\operatorname{det} A^{(k)}\right|$ $=\left|\operatorname{det} A^{k}\right|$. Then $C$ is the matrix of the first $p$ columns of $B A^{(k)}$. Expanding $\operatorname{det} B A^{(k)}$ by elements of the last row, then expanding each of the $(n-1)$ th order determinants so obtained by elements of their last rows and repeating the process as of ten as necessary, we obtain

$$
|\operatorname{det} B|\left|\operatorname{det} A^{k}\right| \leqq \sum_{j}\left|\operatorname{det} C^{j}\right| \beta^{n-p}(n-p) !
$$

On summing this inequality over all submatrices $A^{k}$ and rewriting, we obtain the inequality (3.1).

The estimate of this lemma is certainly very poor but it is sufficient for what follows.

Theorem 2.2. Let $S=A H_{p}$ (where $A^{*} A=I$ on $|z|=1$ ) be a translation invariant subspace of $H_{n}$ of $\operatorname{dim} p$. Then $S$ is i.c. if and only if there exists an $\epsilon>0$ such that

$$
\sum\left|\operatorname{det} A^{k}(z)\right| \geqq \epsilon \quad \text { for all } z \in \Delta,
$$

where $\left\{A^{j}(z)\right\}$ is the set of all $p \times p$ submatrices of the $n \times p$ matrix $A(z)$.

Proof. Suppose that $S$ is i.c. By Theorem 2.1 there exists a matrix $B(z)$ of elements of $H^{\infty}$ such that, for all $z \in \Delta,|\operatorname{det} B(z)| \geqq \alpha>0$, $\left|B_{i j}(z)\right| \leqq \beta$ and $(B(z) A(z))_{i j}=\delta_{i j}$ for $i \leqq p$, and zero otherwise. By Lemma 2.2 the inequality (3.2) now holds for some $\epsilon$.

Conversely suppose that (3.2) is valid. Assume first that $p=1$, i.e. $A(z)=\left\{A_{j}(z)\right\}, j=1, \cdots, n$, and $\sum\left|A_{j}(z)\right| \geqq \epsilon$ in $\Delta$. By the Corona Theorem [1] there exist functions $\theta_{j}(z) \in H^{\infty}$ such that $\sum_{j} \theta_{j} A_{j}=1$ in 
$\Delta$. Let $P: H_{n} \rightarrow H_{n}$ be the matrix operator given by $P_{i j}=A_{i} \theta_{j}, i, j$ $=1, \cdots, n$, then $P$ is a projection onto $S$ which commutes with $z$. Thus $S$ is i.c. as required.

Now assume the theorem true for all $S$ with $\operatorname{dim} S<p(p>1)$. If (3.2) holds it is easily checked that for some $\epsilon>0, \sum_{j}\left|A_{j p}(z)\right| \geqq \epsilon$ and $\sum\left|\operatorname{det} B^{k}(z)\right| \geqq \epsilon$ in $\Delta$, where $B$ is the $n \times(p-1)$ matrix obtained from $A$ by deleting the last column. By assumption then, both $S^{\prime}$ $=\left\{\left\{A_{j p} u\right\} \mid u \in H^{2}\right\}$ and $S^{\prime \prime}=B H_{p-1}$ are i.c. and $S=S^{\prime}+S^{\prime \prime}$. Let $D^{-1}$ be the isomorphism of Theorem 2.1 such that $S^{\prime \prime}=D^{-1} P_{p-1} H_{n}$ then with the proper identifications, $(D B)_{i j}=\delta_{i j}$ if $i \leqq p-1$ and zero otherwise while $(D A)_{i j}=\delta_{i j}$ if $i \leqq p-1$, and zero if $i>p-1 \& j<p-1$, or $j>p$. But applying Lemma 2.2 we have that

$$
\sum_{k}\left|\operatorname{det}(D A)^{k}\right| \geqq \epsilon^{\prime}
$$

for some $\epsilon^{\prime}$. However now

$$
\sum_{k}\left|\operatorname{det}(D A)^{k}\right|=\sum_{j=p}^{n}\left|(D A)_{j p}\right| .
$$

Thus $S_{1}=\left\{\left\{(D A)_{j p} u\right\}_{j=p}^{n} \mid u \in H^{2}\right\}$ is an i.c. subspace of $H_{n-p+1}$ of $\operatorname{dim} 1$. Also in this coordinate system $S^{\prime \prime}=H_{p-1}$. But now $S=S^{\prime}+S^{\prime \prime}$ $=S_{1} \oplus H_{p-1}$ and since $H_{n-p+1} \cap H_{p-1}=0$, and $S_{1}$ is an i.c. subspace of $H_{n-p+1}, S$ is itself i.c. Since the property of being i.c. is rather trivially preserved under linear isomorphisms of $H_{n}$ which commute with $z, S$ in the original coordinate system is i.c. as required.

The last paragraph of the proof of Theorem 2.2 yields with a little modification a necessary and sufficient condition that the sum of two i.c. subspaces be i.c. We omit the proof but state the result.

Theorem 2.2'. If $S=A H_{p}$ and $S^{\prime}=A^{\prime} H_{q}$ are i.c. subspaces of $\operatorname{dim} p$ and $q$ respectively, then a necessary and sufficient condition that $S+S^{\prime}$ be i.c. of $\operatorname{dim} p+q$ is that

$$
\sum\left|\operatorname{det} C^{k}\right| \geqq \epsilon>0 \quad \text { in } \Delta
$$

where $\left\{C^{k}\right\}$ is the set of $(p+q) \times(p+q)$ submatrices of the $n \times(p+q)$ matrix $C$ whose columns are those of $A$ and those of $A^{\prime}$.

As a statement about matrices of elements from $H^{\infty}$, Theorem 2.2 has an interesting interpretation. Observing that the fact $A^{*} A=I$ was not used in the proof but appeared merely because we chose to use the Lax representation for $S$, we have on combining Theorems 2.1 and 2.2 , 
CoRollary. If $A$ is an $n \times p$ matrix of elements from $H^{\infty}$ then there exists a nonsingular matrix $B$ of elements from $H^{\infty}$ such that $(B A)_{i j}=\delta_{i j}$ if $i \leqq p$ and zero otherwise if and only if $\sum\left|\operatorname{det} A^{k}\right| \geqq \epsilon>0$ in $\Delta$.

A particular case of this Corollary (essentially the case $n=r p, r$ an integer, but without showing $B$ may be chosen nonsingular) has been given by Fuhrmann [2, Theorem 3.1].

\section{REFERENCES}

1. L. Carleson, Interpolation by bounded analytic functions and the corona problem, Ann. of Math. (2) 76 (1962), 547-559.

2. P. A. Fuhrmann, On the corona theorem and its application to spectral problems in Hilbert space, Trans. Amer. Math. Soc. 132 (1968), 55-66.

3. P. R. Halmos, Shifts on Hilbert spaces, Crelle's Journal 208 (1961), 102-112.

4. - A Hilbert space problem book, Van Nostrand, Princeton, N. J., 1967.

5. H. Helson, Lectures on invariant subspaces, Academic Press, New York, 1964.

6. P. D. Lax, Translation invariant spaces, Acta. Math. 101 (1959), 163-178.

University of California, Los Angeles 